$>$ Lors de l'accumulation de protéines mal conformées dans le réticulum endoplasmique (RE), une réponse adaptative nommée UPR (unfolded protein response) est induite afin de protéger la cellule contre ce stress. Chez les métazoaires, cette réponse est assurée par trois protéines transmembranaires du RE : PERK (PKR-related endoplasmic reticulum kinase), ATF6 (activating transcription factor 6) et IREI (inositol requiring enzyme 1). Parmi ces trois protéines, seule IREl est conservée au cours de l'évolution. IREl est une protéine transmembranaire de type I qui possède deux activités enzymatiques, sérine/ thréonine kinase et endoribonucléasique. Les structures cristallines des domaines luminal et cytosolique de la protéine IREl obtenues chez $S$. cerevisiae ont permis de mieux comprendre son mode de fonctionnement. Néanmoins, de nombreuses zones d'ombres subsistent concernant les mécanismes de régulation d'IREl ainsi que les différentes voies de signalisation induites en réponse à son activation. Cette revue propose une synthèse des connaissances actuelles à propos de la protéine IREl et analyse particulièrement le rôle d'IREl dans les mécanismes physiologiques et physiopathologiques. <

\section{Stress du réticulum endoplasmique}

\section{Une réponse pour éviter le pIRE}

Marion Bouchecareilh, Eric Chevet

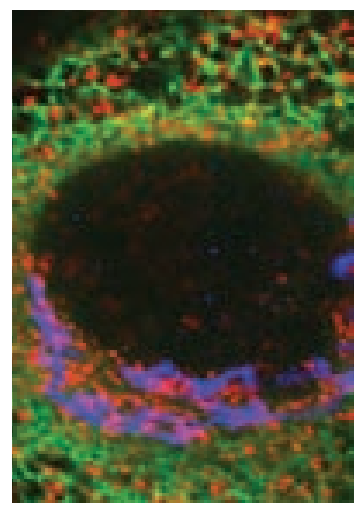

Avenir Inserm U889, Université Bordeaux 2, 146, rue Léo Saignat, 33076 Bordeaux, France. eric.chevet@u-bordeaux2.fr

charge protéique observée dans le RE via un programme transcriptionnel spécifique, permettant une augmentation de l'expression de protéines chaperons ou de protéines impliquées dans la machine de dégradation des protéines associée au RE ( $E R A D)$. Si l'homéostasie du RE n'est pas rétablie, alors la deuxième phase du programme conduisant à l'apoptose se déclenche [44]. Cette réponse adaptative intégrée est contrôlée principalement par 3 protéines transmembranaires résidant dans le RE: PERK (PKR-related endoplasmic reticulum kinase), ATF6 (activating transcription factor 6) et enfin IREI (inositol requiring enzyme 1). La première de ces 3 protéines à avoir été mise en évidence est IREl. Elle est la seule à être présente chez les Eucaryotes, de la levure à l'homme. IREl représente donc le bras le plus conservé de l'UPR. L'objetif de cette revue est de faire le point sur les différentes connaissances acquises sur la protéine IREl au cours des dernières années.

\section{IRE1 - généralités}

IREla ou ERNI (endoplasmic reticulum to nucleus signalling 1) est une protéine transmembranaire de type l. Cette protéine de 110 kDa ou 977 acides aminés (homo sapiens) possède deux activités enzymatiques, sérine/thréonine kinase et endoribonucléasique, dans son domaine cytosolique. Chez les mammifères, contrairement à la levure, il existe deux isoformes $\alpha$ et $\beta$ de la protéine IREI (ERN1 et ERN2, respectivement). Ces deux isoformes ne sont pas codées par le même gène. À titre 
d'exemple, chez l'homme, le gène codant pour IREl $\alpha$ est situé sur le chromosome 17 (17q24.2) alors que le gène codant pour IREl $\beta$ est situé sur le chromosome 16 (16p12.2). La protéine IREl a été identifiée dans les années 1990 chez $S$. cerevisiae. Elle est activée en réponse à la baisse de concentration en inositol et conduit à la transcription de gènes impliqués dans la biosynthèse de ce lipide comme l'inositol-1-phosphate synthase (INOI) [1]. Le gène codant pour IREl a été séquencé dans de nombreuses espèces (Figure I) et la séquence partage $50 \%$ d'identité dans le domaine kinase entre l'homme, $S$. cerevisiae et $C$. elegans.

\section{Structure d'IREI}

\section{Le domaine luminal}

Le domaine luminal de IREl (Figure 2 ; partie supérieure du schéma) est composé d'un peptide signal de ciblage dans le RE et d'un domaine de dimérisation. Des mutations dans ce domaine abolissent sa capacité de dimérisation et les changements conformationnels transmis au domaine cytosolique via le segment transmembranaire. La structure de ce domaine suggère que l'homodimérisation décrite pourrait être en fait une oligomérisation [2]. IREl possède aussi un domaine de liaison à la protéine chaperon BiP. Ce chaperon est impliqué dans le maintien de la protéine IREl dans un état monomérique et inactif. Lors d'un stress du RE, BiP se dissocierait d'IREl pour prendre en charge les protéines mal conformées. IREl ainsi libérée pourrait alors se dimériser et s'activer [3]. Néanmoins, des travaux récents réalisés chez S. cerevisiae montrent que la délétion du domaine de liaison à BiP ne rend pas Irelp constitutivement active et n'inhibe pas la régulation d'Irelp que des protéines mal conformées soient ou non présentes dans le RE [4]. De plus, la structure du domaine luminal présente des similitudes avec le complexe majeur d'histocompatibilité de classe 1,

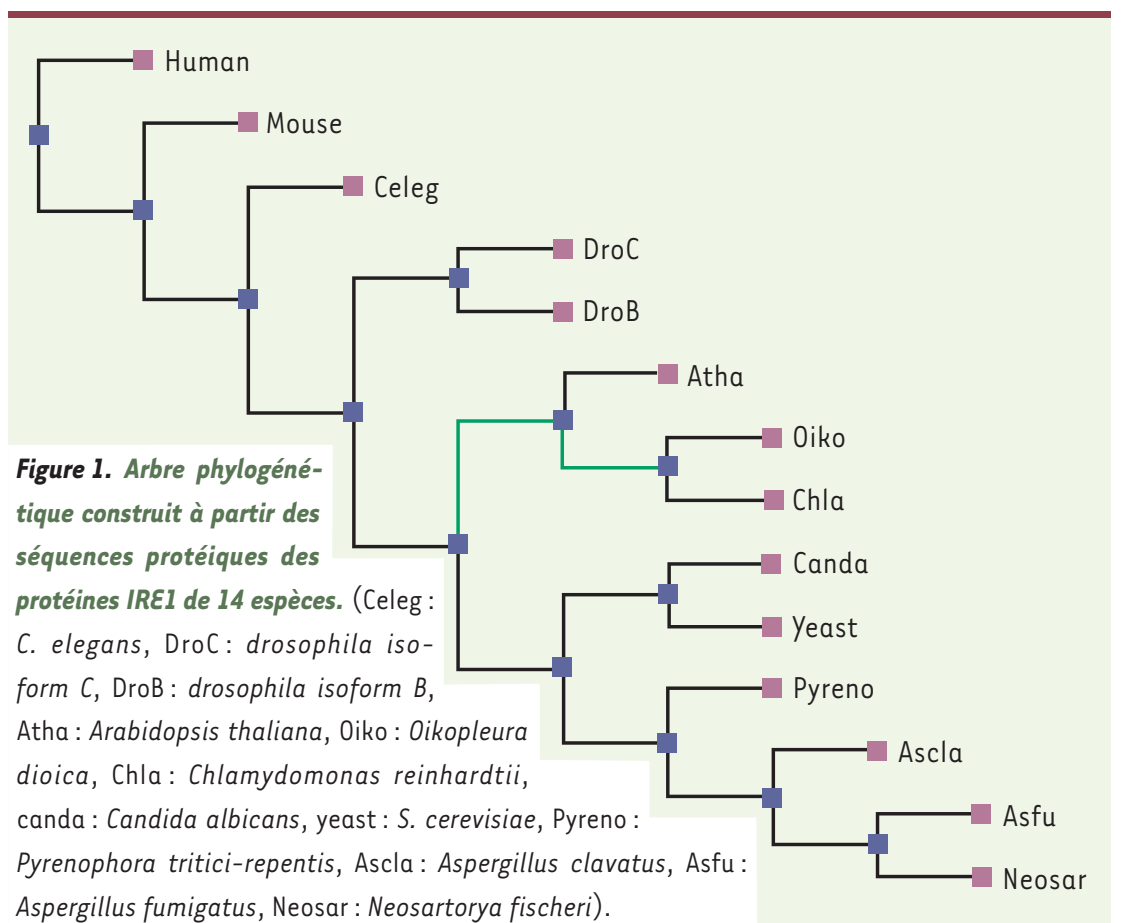

ce qui suggère que la protéine IREl pourrait directement interagir avec les protéines mal conformées [2]. Le modèle initial d'association à BiP ne semble donc pas suffisant pour permettre l'activation d'IREl. Une étape supplémentaire, comme la reconnaissance des protéines mal conformées par IREl, pourrait aussi jouer un rôle dans ce mécanisme.

\section{Le domaine cytosolique}

L'oligomérisation du domaine luminal d'IREl permet la juxtaposition des domaines cytosoliques et facilite l'autophosphorylation dans le domaine kinase et l'activation du domaine RNase [5]. La structure du domaine cytosolique d'Irelp (S. cerevisiae) suggère, tout comme celle du domaine luminal, que cette protéine pourrait exister à l'état de dimère en l'absence de stress [5] (Figure 2; partie inférieure du schéma). L'activation pourrait alors résulter de changements conformationnels. La séquence en acides aminés du domaine cytosolique d'Irelp de S. cerevisiae présente $29 \%$ d'identité avec celle du domaine catalytique de la RNase L [6, 45]. Ces deux protéines composent une famille unique possédant un domaine kinase (ou pseudo-kinase) et une activité endoribonucléasique. L'activité RNase de ces deux enzymes est régulée par la dimérisation ainsi que par la liaison à un nucléotide [7] de type adénosine di ou tri-phosphate [8]. Le nucléotide, en se fixant sur le site kinase, permet un changement conformationnel induisant l'activité RNase [5].

\section{Voies de signalisation dépendantes d'IREI}

\section{Chez S. cerevisiae}

IREl est la protéine clé de la réponse UPR chez la levure comme le confirme le knock-out (KO) du gène codant pour IREI qui se traduit par une perte complète de la réponse UPR [9]. Chez $S$. cerevisiae, Irelp participe à l'épissage de l'ARNm codant pour Hacl. Cela inclut deux étapes, le clivage d'un intron de 252 nucléotides par Irelp et la ligation des fragments résultants par la protéine ARNt ligase RgIlp [10]. L'ARNm non épissé codant pour Hacl n'est jamais traduit car l'intron bloque la progression du ribosome sur l'ARNm [10]. En revanche, I'ARNm épissé est traduit et joue son rôle de facteur de transcription en se fixant sur les séquences UPRE (unfolded protein response responsive element) 


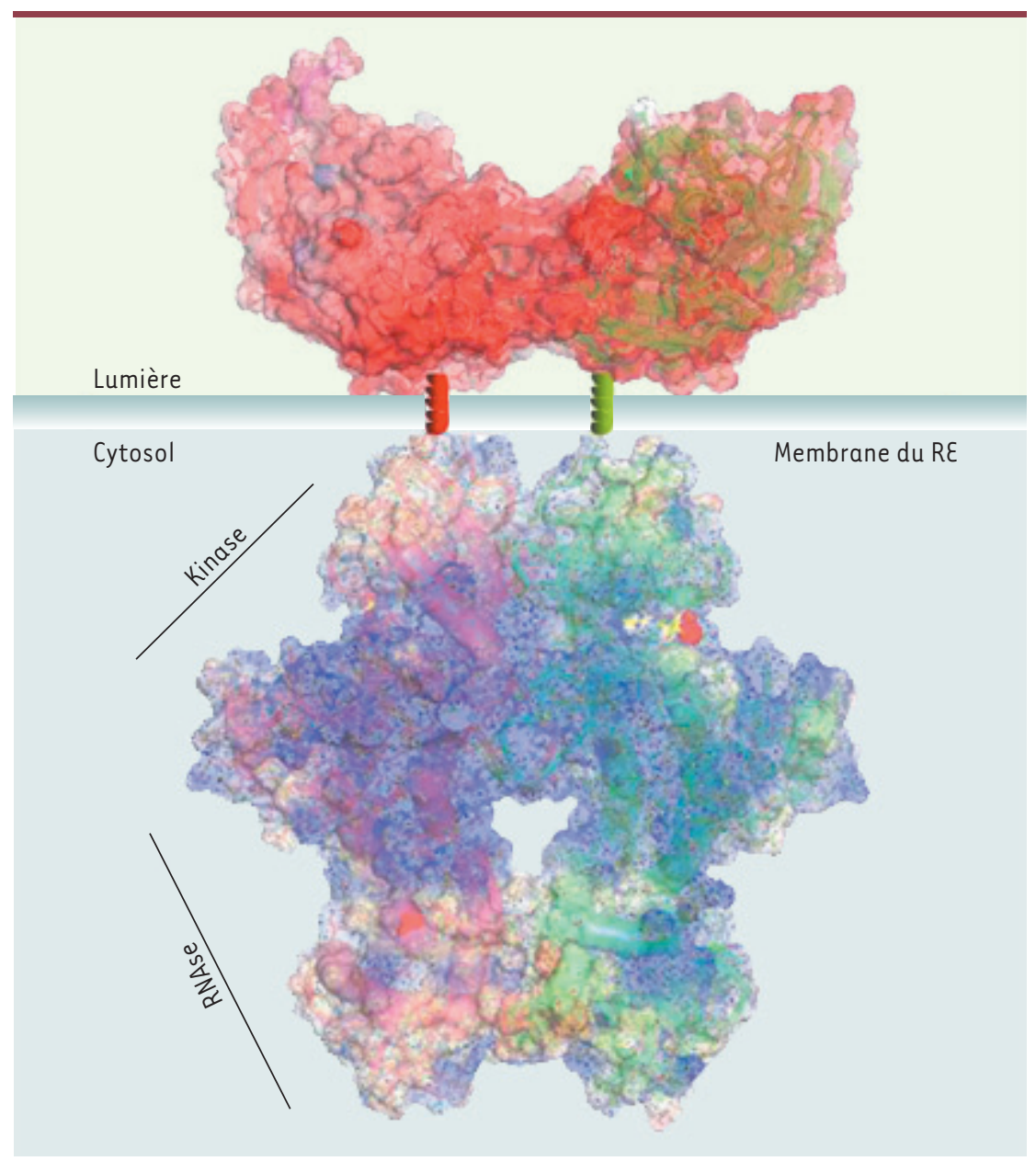

Figure 2. Structure d'IREI sous forme d'homodimère (chaînes $A$ et $B$ ). IREl est composée d'un domaine luminal et d'un domaine cytosolique comprenant deux activités enzymatiques: une activité sérine/thréonine kinase et une activé endoribonucléasique (RNAse). Lors d'un stress du RE, IREl s'homodimérise pour induire les voies de signalisation en aval. Cette représentation a été réalisée à partir des structures cristallines de Irep chez S. cerevisiae. Le potentiel electrostatique des deux régions (luminale et cytosolique) est indiqué en suivant l'échelle (en bas à droite). Les domaines transmembranaires présomptifs des deux chaînes $A$ et $B$ sont schématisés en rouge et vert, respectivement.

voie de signalisation dépendante de l'activité endoribonucléasique de IREI, d'autre part une cascade d'activation de protéine kinases.

La première de ces deux voies de signalisation est impliquée dans l'épissage de I'homologue fonctionnel de Haclp nommé XBPI ( $X$-box binding protein 1$)$ [16]. IREl clive un intron de taille variable selon l'espèce (26 nucléotides chez l'homme) et une ligation des fragments présentes au niveau des promoteurs des gènes cibles spécifiques [11]. L'UPR active la transcription d'environ 381 gènes [12] dont certains codent pour des protéines chaperons (Kar2), des protéines du stress redox (PDIl, ERO1), de l'ERAD (DERl, HRDI) ou encore du métabolisme lipidique (INO1). Irelp est régulée de façon positive par le complexe co-activateur nommé SAGA composé des protéines GCN5, Ada2 et Ada3 et Ada5 et qui joue un rôle essentiel dans la signalisation d'Irelp et la transcription de gènes induite lors de la réponse UPR [13]. Ceci est probablement dû au fait qu'Ada5p interagit directement avec Irelp et que sa présence est requise pour l'épissage de l'ARNm codant pour Hacl [13]. Irelp est également régulée de façon négative par les phosphatases Ptc2p et Dcr2p qui la déphosphorylent. Le KO de PTC2 augmente de 4 fois la réponse UPR et l'épissage de Haclp alors que la surexpression de Ptc2p ou de Dcr2p produit l'effet inverse [14, 15].

\section{Chez les métazoaires}

Au moins deux voies de signalisation majeures sont activées en aval de la protéine IREl. D'une part une produits intervient via une protéine inconnue différente de Rgllp [17] (Figure 3). Cet épissage conduit à un changement du cadre de lecture lors de la traduction de la protéine. XBPI est un facteur de transcription appartenant à la famille ATF/CREB et possède dans la région amino-terminale un domaine leucine zipper qui lui permet de se fixer sur les promoteurs des gènes contenant une séquence $E R S E$ ( $E R$ stressresponse element) ou UPRE. L'activation de l'axe de signalisation IREl/ $X B P$ induit l'expression d'un certain nombre de gènes cibles de I'UPR comme des gènes codant pour des protéines de la voie ERAD (EDEM, HRD1) ou des chaperons (BiP) [16]. À l'inverse de Haclp, les formes épissées et non épissées de XBPl sont traduites. La protéine traduite à partir de l'ARNm non épissé de XBPI inhibe la réponse UPR en se fixant sur la protéine traduite à partir de l'ARNm XBPI épissé. Ce complexe est alors dégradé par le protéasome. La protéine traduite à partir de l'ARNm non épissé de XBPI représente donc un régulateur négatif de la réponse UPR [18]. II semble enfin que la protéine IREl soit I'unique endoribonucléase responsable du clivage de l'ARNm codant pour XBP1. Par conséquent, l'axe de signalisation IREl/XBPI peut être considéré comme indissociable. De plus, outre l'ARNm XBPl, IREl peut cliver/dégrader spécifiquement certains ARNm (SPARC, CD59) [19], I'ARNm codant pour l'insuline [20] ou certains ARNm codant pour des protéines de l'horloge circadienne ( 0 . Pluquet et $\varepsilon$. Chevet, résultats 
non publiés). D'autre part IREl peut réguler son expression en clivant son propre ARNm [8]. Ces régulations post-transcriptionnelles dépendantes de la protéine IREl pourraient se produire indépendamment de l'épissage de XBPI.

Hormis son activité endoribonucléasique, IREl s'associe à de nombreux partenaires par l'intermédiaire de son domaine cytosolique pour induire différentes voies de signalisation impliquant des protéines kinases. TRAF2 (TNF receptor-associated factor), une protéine adaptatrice, s'associe au domaine kinase d'IREl. Le complexe IREl/TRAF2 interagit alors avec ASKl (apoptosis signal-regulating kinase 1) pour activer la JNK, c-Jun N-terminal kinase [21]. La protéine adaptatrice $\mathrm{NCKl}$ interagit via son domaine $\mathrm{SH} 3$ avec la région carboxy-terminale d'IREl en l'absence de stress. Lors d'un stress du RE, NCKl se dissocie d'IREl pour permettre l'activation de la voie des ERK (extra cellular regulated kinases) [22]. Enfin IREl interagit avec la protéine chaperon HSP90 qui se fixe sur son domaine cytosolique et en permet la stabilisation [23].

\section{IRE1, ATF6 et PERK}

Des connexions directes entre les différents senseurs du stress UPR n'ont pas encore été établies, néanmoins il existe une coordination entre les différents axes de la réponse UPR. L'exemple le plus représentatif de cette coordination intervient au cours de l'activation de ATF6 qui conduit à la transcription du gène codant pour XBPl. Cet ARNm est alors épissé par IREl. D'autre part, des travaux ont montré que les protéines ATF6 et XBPI co-immunoprécipitent, ce qui suggère que ces deux facteurs de transcription peuvent former un hétérodimère in vivo [24]. De plus certains gènes induits par XBPl régulent de façon négative l'activité de PERK [25]. II en est de même pour la protéine adaptatrice NCKI : non seulement celle-ci interagit avec IREl, mais elle est impliquée dans l'atténuation de la voie PERK en favorisant la phosphorylation d'elF2 $\alpha$ [26].

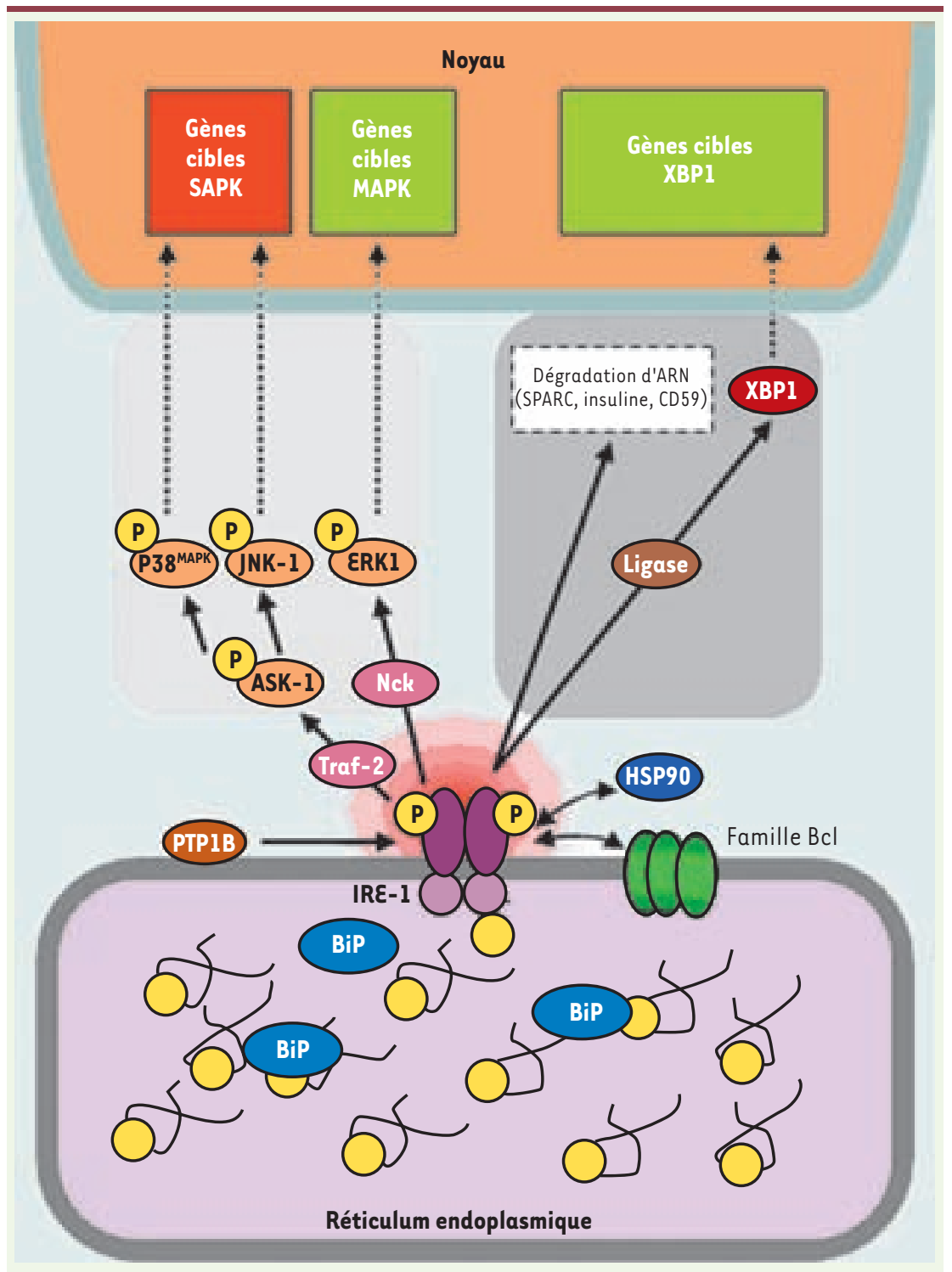

\section{Survie et apoptose}

La réponse UPR dans laquelle intervient IREl est avant tout une réponse cytoprotective qui permet à la cellule de s'adapter à un stress protéotoxique. Néanmoins si ce stress devient trop sévère, la cellule ne peut pas y faire face et entre alors en apoptose. IREl intervient dans la régulation de cette balance entre cytoprotection et apoptose. En effet, bien que les voies de signalisation conduisant à ces deux phénotypes soient activées simul-

Figure 3. Représentation schématique des voies de signalisation dépendantes de la protéine IRE1. Les protéines mal conformées sont indiquées par des symboles jaunes dans la lumière du RE. Les protéines chaperons sont indiquées en bleu, les kinases en orange, les adaptateurs en rose, les facteurs de transcription en bordeaux, les molécules pro-apoptotiques en vert, les autres enzymes impliquées en brun (ligases) ou rouge (phosphatase). Deux axes de signalisation majeurs sont activés en aval de la protéine IREl (carrés gris clair: kinases et gris foncé : régulations post-transcriptionnelles comme l'épissage de XBPI ou la dégradation d'ARN cibles, carré pointillé). Les gènes cibles activés dans le noyau en réponse à ces voies de signalisation sont indiqués dans les boîtes vertes (pro-survie) ou rouge (proapoptotiques). 
tanément, le résultat final intègre la durée et l'intensité d'activation de chacune de ces voies de signalisation pour conduire à la mort ou à la survie de la cellule.

\section{Survie}

L'autophagie, considérée comme un processus de survie, est induite par des agents qui perturbent directement l'homéostasie du RE [27]. IREl, via l'induction de la voie IREl/TRAF2/JNK, semble jouer un rôle crucial dans l'induction d'autophagosomes et favoriserait donc la survie cellulaire. JNK pourrait également induire une inhibition de la voie mTOR (target of rapamycine) qui, à l'inverse d'IREl, inhibe la formation d'autophagosomes [28]. Néanmoins le rôle d'IREl dans l'autophagie n'est pas exclusif puisque dans certains cas particulier (polyglutamination), la voie PERK/elF2 $\alpha$ peut également induire la formation d'autophagosomes [29]. L'épissage de XBPl est un événement transitoire qui disparaît après approximativement $16 \mathrm{~h}$ lors d'un stress du RE. Néanmoins si l'épissage de XBPl, et donc l'activation de la voie IREl/XBPl, sont maintenus plus de $48 \mathrm{~h}$, le nombre de cellules apoptotiques diminue. II semble donc que la voie IREl/XBPl joue un rôle dans le maintien de la survie cellulaire au cours du stress du RE [30].

\section{Apoptose}

IREl, via TRAF2, recrute et active la protéine ASKl qui à son tour active JNK. Cela induit la mort cellulaire par apoptose [21]. De plus, TRAF2 une fois liée à IREl pourrait également interagir avec la procaspase 12 . Cela permet l'homodimérisation et le clivage de cette procaspase dans des systèmes expérimentaux dans lesquels elle est activable. La caspase 12 active pourrait alors actionner une cascade protéolytique impliquant la caspase-9 et la caspase-3 [31]. Les protéines proapoptotiques BAX/BAK interagissent avec le domaine cytosolique d'IREl. Cette interaction peut conduire à l'induction de l'apoptose et semble nécessaire à l'activité d'IRE1 [32]. Enfin, dans des cellules déficientes pour la protéine phosphatase PTPIB, l'épissage de XBP1 ainsi que la phosphorylation de JNK sont diminués. Ces cellules sont également plus résistantes à l'apoptose induite lors d'un stress du RE [33].

\section{Rôles physiologiques et physiopathogiques d'IREI}

Contrairement aux autres senseurs de la réponse UPR, IREl est le seul dont la délétion conduit à une létalité embryonnaire qui survient entre les stades $\varepsilon 9,5$ et $\varepsilon 11,5$ [21]. Il en est de même pour XBPl, le KO du gène XBP-1 induit une létalité embryonnaire au stade E14.5 [34].
La voie IREl/XBPl représente donc un axe de signalisation essentiel au cours du développement. IREl intervient également dans de nombreux processus physiologiques en particulier dans les cellules ayant un besoin accru en production et sécrétion de protéines. C'est le cas des cellules acinaires pancréatiques qui sécrètent des enzymes digestives ou encore des cellules plasmocytaires qui sécrètent les immunoglobulines. En effet XBPl (et par extension l'épissage de l'ARNm codant pour cette protéine par $\mid R \varepsilon l$ ) joue un rôle prépondérant dans la synthèse d'immunoglobulines en induisant la différenciation des cellules plasmocytaires. L'inactivation d'XBPI induit une inhibition de la biosynthèse lipidique. La taille du RE est alors peu développée ce qui entraîne une diminution de la production de protéines et de la quantité de protéines sécrétées et peut aboutir à la mort des cellules plasmocytaires [35]. L'axe de signalisation impliquant IREl et XBPI intervient également dans la régulation de la lipogenèse dans le foie. L'inhibition de l'épissage de XBPl ou de son expression induit une diminution de la synthèse de lipides au niveau hépatique ce qui conduit à la diminution des triglycérides, des acides gras libres et du cholestérol dans le sérum [36]. Si la protéine IREl intervient dans un certain nombre de processus physiologiques, elle jouerait également un rôle crucial dans de nombreuses pathologies. En effet des études ont montré que l'inhibition de la signalisation de la protéine IREl induisait une diminution de la croissance et de l'angiogenèse tumorales. IREl permettrait l'adaptation des cellules tumorales à leur environnement ischémique en induisant entre autres la production de facteurs pro-angiogéniques tel que le VEGF-A (vascular endothelial growth factor) [37]. II en est de même pour XBP1, qui est également impliqué dans le développement tumoral [38, 39]. D'autre part, des mutations somatiques non silencieuses du gène IREI sont fréquemment observées dans certains cancers humains et pourraient conduire à des gains ou pertes de fonctions [40]. IREl intervient également dans les processus de mort cellulaire au cours de maladies neurodégénératives. Ainsi, dans la maladie d'Alzheimer et plus particulièrement sa forme familiale (FAD), les produits de trois gènes ont été identifiés comme étant responsables de cette pathologie: l'amyloid precursor protein (APP), et les présénilines 1 et 2 (PS1; PS2), les mutations de la PS1 étant les plus fréquemment rencontrées chez les patients atteint d'une FAD [41]. De plus, des études in vitro ont mis en évidence que les cellules exprimant une forme mutée de PSI sont plus sensibles aux stimulus apoptiques induits par le stress du RE. Cela serait du à l'interaction directe entre la PSI et IREl. PSI mutée inhiberait la phosphorylation d'IREl induite lors d'un stress du RE. Néanmoins, PSI ne cible pas spécifiquement IREl puisqu'elle inhibe également l'activation des autres senseurs de l'UPR, ATF6 et PERK [41]. Enfin IREl a été identifiée comme jouant un rôle important dans les phénomènes d'insulino-résistances périphérique. En effet, le stress du RE et en particulier l'altération de l'axe de signalisation IREl/XBPI, ont été corrélés à une signalisation déficiente du récepteur à l'insuline dans le foie [42]. D'autre part, les travaux de Lipson et al. ont mis en évidence que les cellules $\beta$ pancréatiques soumises à une hyperglycémie chronique induisent un stress du RE et une augmentation de la phosphorylation d'IREl. Cette augmentation a pour conséquence une inhibition de la synthèse/expression d'insuline [20,42]. 


\section{Conclusion}

IREl est une protéine hautement conservée au cours de l'évolution. IREl est activée lors d'un stress du RE et représente l'une des protéines clés de la réponse UPR. Tout comme les récepteurs à activité tyrosine kinase, IREl s'active par dimérisation ce qui induit son auto-transphophorylation et son activité RNasique. IREl joue un rôle critique dans les processus d'apoptose et de survie au cours du stress du RE observés dans des conditions physiologiques ou physiopathologiques. L'identification des mécanismes de régulation naturels de cette protéine et des voies de signalisation issues de son activation représente par conséquent une voie de recherche à explorer. De plus, la modulation pharmacologique de l'activité biologique d'IREl pourrait alors constituer un nouveau type d'approche thérapeutique dans de nombreuses pathologies. $\diamond$

\section{GLOSSAIRE}

APP : Amyloid precursor protein

ASK1 : Apoptosis signal-regulating kinase 1

ATF6: Activating transcription factor

CD59: Protéine liée aux lipides membranaires, par une ancre GPI (glycophosphoinositol), qui empêche la liaison de complexes du complément à la membrane

DER1 : Degradation in endoplasmic reticulum protein 1

ERAD : Endoplasmic reticulum-associated degradation (ERAD)

ERK : Extracellular regulated kinases

ERN1 : Endoplasmic reticulum to nucleus signalling 1

ER01 : Endoplasmic Reticulum Oxidoreductin 1-L

ERS : $\varepsilon R$ stress-response element

HRD1 : $\varepsilon R A D$-associated $\varepsilon 3$ ubiquitin-protein ligase

HSP90 : Heat shock protein 90

IN01: Inositol-1-phosphate synthase

IRE1 : Inositol requiring enzyme I

JNK : c-Jun $\mathrm{N}$-terminal kinase

mTOR : target of rapamycine

NCK1 : Non-catalytic region of tyrosine kinase adaptor protein 1

PDI1 : Protein disulfure isomerase de $59 \mathrm{kDa}$

PTP1B : A nontransmembrane tyrosine phosphatase localisée dans le RE

PERK : PKR-related endoplasmic reticulum kinase

SAPK/JNK : stress-activated protein kinase/c-Jun $\mathrm{NH}_{2}$-terminal kinase

SPARC: Secreted protein, acidic, cysteine-rich (osteonectin)

TRAF2 : TNF-receptor associated factor

UPRE : Unfolded protein response responsive elements

VEGF-A : Vascular endothelial growth factor type $A$

XBP1 : X-box binding protein 1

\section{SUMMARY}

Endoplasmic reticulum stress: light my fIRE

Upon accumulation of misfolded proteins in the lumen of the endoplasmic reticulum $(\varepsilon R)$, a specific adaptive response, named the Unfolded Protein Response (UPR) is activated in order to protect cells from this stress. In metazoans, the UPR is mediated by three transmembrane $\varepsilon R$ resident proteins: PERK, ATF6 and IREl. Among these, only IREl is found to be conserved from yeasts to mammals. IREl is a type I transmembrane protein which bears two enzymatic activities serine/threonine kinase and endoribonuclease. Crystal structures of $S$. cerevisiae luminal and cytosolic domains allowed a better understanding of its activation mode. However, IREl regulatory mechanisms and IREl-dependent signalling pathways still remain to be fully explored. This review will present current knowledge on IREl protein and focus on its roles in physiological and pathophysiological processes. $\diamond$

\section{REMERCIEMENTS}

Ce travail a été réalisé avec le soutien de la Fondation pour la Recherche Médicale, de l'Institut National du Cancer, d'un programme Avenir (Inserm) et d'un International Reintegration grant Marie Curie à E.C. M.B. est soutenue par une bourse de thèse du Conseil Régional d'Aquitaine.

\section{RÉFÉRENCES}

1. Nikawa J, Yamashita S. IREl encodes a putative protein kinase containing a membrane-spanning domain and is required for inositol phototrophy in Saccharomyces cerevisiae. Mol Microbiol 1992 ; 6 : 1441-6.

2. Credle JJ, Finer-Moore JS, Papa FR, et al. On the mechanism of sensing unfolded protein in the endoplasmic reticulum. Proc Natl Acad Sci USA 2005 ; $102: 18773-84$

3. Bertolotti A, Zhang $Y$, Hendershot $L M$, et al. Dynamic interaction of BiP and $\varepsilon R$ stress transducers in the unfolded-protein response. Nat Cell Biol 2000 ; $2: 326-32$.

4. Kimata $Y$, Oikawa D, Shimizu $Y$, et al. A role for BiP as an adjustor for the endoplasmic reticulum stress-sensing protein Irel.J Cell Biol 2004 ; $167: 445-56$.

5. Lee KP, Dey M, Neculai D, et al. Structure of the dual enzyme Irel reveals the basis for catalysis and regulation in nonconventional RNA splicing. Cell 2008; $132: 89-100$.

6. Bork P, Sander C. A hybrid protein kinase-RNase in an interferon-induced pathway? FEBS Lett 1993 ; $334: 149-52$.

7. Zhou A, Nie H, Silverman RH. Analysis and origins of the human and mouse RNase L genes: mediators of interferon action. Mamm Genome 2000 ; 11 : 989-92.

8. Tirasophon W, Welihinda AA, Kaufman RJ. A stress response pathway from the endoplasmic reticulum to the nucleus requires a novel bifunctional protein kinase/endoribonuclease (Irelp) in mammalian cells. Genes Dev 1998 ; 12 : 1812-24.

9. Urano F, Bertolotti A, Ron D. IREl and efferent signaling from the endoplasmic reticulum. J Cell Sci 2000 ; 113 : 3697-702.

10. Sidrauski C, Walter P. The transmembrane kinase Irelp is a site-specific endonuclease that initiates mRNA splicing in the unfolded protein response. Cell $1997 ; 90: 1031-9$.

11. Schroder M, Clark R, Kaufman RJ. IREl- and HACl-independent transcriptional regulation in the unfolded protein response of yeast. Mol Microbiol 2003; 49: 59l-606.

12. Travers KJ, Patil CK, Wodicka L, et al. Functional and genomic analyses reveal an essential coordination between the unfolded protein response and $\varepsilon R-$ associated degradation. Cell $2000 ; 101: 249-58$.

13. Welihinda AA, Tirasophon W, Kaufman RJ. The transcriptional co-activator ADA5 is required for $\mathrm{HACl}$ mRNA processing in vivo. J Biol Chem 2000; $275: 3377-81$.

14. Welihinda AA, Tirasophon W, Green SR, Kaufman RJ. Protein serine/threonine phosphatase Ptc2p negatively regulates the unfolded-protein response by dephosphorylating Irelp kinase. Mol Cell Biol 1998; 18 : 1967-77.

15. Guo J, Polymenis M. Dcr2 targets Irel and downregulates the unfolded protein response in Saccharomyces cerevisiae. EMBO Rep 2006 ; 7 : 1124-7.

16. Calfon M, Zeng H, Urano F, et al. IREl couples endoplasmic reticulum load to secretory capacity by processing the XBP-1 mRNA. Nature $2002 ; 415$ : 92-6.

17. Harding HP, Lackey JG, Hsu HC, et al. An intact unfolded protein response in Trptl knockout mice reveals phylogenic divergence in pathways for RNA ligation. RNA 2008 ; $14: 225-32$. 
18. Yoshida H, Oku M, Suzuki M, Mori K. pXBPl(U) encoded in XBPl pre-mRNA negatively regulates unfolded protein response activator $\mathrm{pXBP1}(\mathrm{S})$ in mammalian ER stress response. J Cell Biol 2006; $172: 565-75$

19. Hollien J, Weissman JS. Decay of endoplasmic reticulum-localized mRNAs during the unfolded protein response. Science $2006 ; 313: 104-7$.

20. Lipson KL, Ghosh R, Urano F. The role of IRElalpha in the degradation of insulin mRNA in pancreatic beta-cells. PLOS ONE 2008 ; 3 : el648.

21. Urano F, Wang $X$, Bertolotti $A$, et al. Coupling of stress in the $\varepsilon R$ to activation of JNK protein kinases by transmembrane protein kinase IREl. Science 2000 ; $287: 664-6$.

22. Nguyen DT, Kebache $S$, Fazel $A$, et al. Nck-dependent activation of extracellular signal-regulated kinase- 1 and regulation of cell survival during endoplasmic reticulum stress. Mol Biol Cell 2004 ; 15 : 4248-60.

23. Marcu MG, Doyle M, Bertolotti A, et al. Heat shock protein 90 modulates the unfolded protein response by stabilizing IRElalpha. Mol Cell Biol 2002; $22: 8506-13$.

24. Yamamoto K, Sato T, Matsui T, et al. Transcriptional induction of mammalian $\varepsilon R$ quality control proteins is mediated by single or combined action of ATF6alpha and XBP1. Dev Cell $2007 ; 13: 365-76$.

25. Yan W, Frank CL, Korth MJ, et al. Control of PERK elF2alpha kinase activity by the endoplasmic reticulum stress-induced molecular chaperone P58IPK. Proc Natl Acad Sci USA 2002 ; 99 : 15920-5.

26. Kebache $S$, Cardin $\varepsilon$, Nguyen DT, et al. Nck-1 antagonizes the endoplasmic reticulum stress-induced inhibition of translation. J Biol Chem 2004 ; 279: $9662-71$

27. Ding WX, Yin XM. Sorting, recognition and activation of the misfolded protein degradation pathways through macroautophagy and the proteasome. Autophagy $2008 ; 4: 141-50$.

28. Ding WX, Ni HM, Gao W, et al. Linking of autophagy to ubiquitin-proteasome system is important for the regulation of endoplasmic reticulum stress and cell viability. Am J Pathol $2007 ; 171: 513-24$

29. Kouroku Y, Fujita $\varepsilon$, Tanida I, et al. ER stress (PERK/elF2alpha phosphorylation) mediates the polyglutamine-induced LC3 conversion, an essential step for autophagy formation. Cell Death Differ 2007 ; $14: 230-9$.

30. Lin JH, Li H, Yasumura D, et al. IREl signaling affects cell fate during the unfolded protein response. Science $2007 ; 318: 944-9$.
31. Yoneda T, Imaizumi K, Oono K, et al. Activation of caspase-12, an endoplastic reticulum (ER) resident caspase, through tumor necrosis factor receptor-associated factor 2-dependent mechanism in response to the $\varepsilon R$ stress. J Biol Chem $2001 ; 276: 13935-40$.

32. Hetz C, Bernasconi P, Fisher J, et al. Proapoptotic BAX and BAK modulate the unfolded protein response by a direct interaction with IRElalpha. Science $2006 ; 312$ : 572-6.

33. Gu F, Nguyen DT, Stuible M, et al. Protein-tyrosine phosphatase $1 B$ potentiates IREl signaling during endoplasmic reticulum stress. J Biol Chem $2004 ; 279$ : 49689-93.

34. Masaki T, Yoshida M, Noguchi S. Targeted disruption of CRE-binding factor TREB5 gene leads to cellular necrosis in cardiac myocytes at the embryonic stage. Biochem Biophys Res Commun 1999 ; $261: 350-6$.

35. Iwakoshi NN, Lee AH, Vallabhajosyula P, et al. Plasma cell differentiation and the unfolded protein response intersect at the transcription factor XBP-1. Nat Immunol 2003; $4: 321-9$.

36. Lee AH, Scapa $E F$, Cohen DE, Glimcher LH. Regulation of hepatic lipogenesis by the transcription factor XBP1. Science $2008 ; 320: 1492-6$.

37. Drogat $B$, Auguste $P$, Nguyen DT, et al. IREl signaling is essential for ischemia-induced vascular endothelial growth factor-A expression and contributes to angiogenesis and tumor growth in vivo. Cancer Res $2007 ; 67: 6700-7$.

38. Romero-Ramirez L, Cao H, Nelson D, et al. XBP1 is essential for survival under hypoxic conditions and is required for tumor growth. Cancer Res $2004 ; 64: 5943-7$.

39. Carrasco DR, Sukhdeo K, Protopopova M, et al. The differentiation and stress response factor XBP1 drives multiple myeloma pathogenesis. Cancer Cell $2007 ; 11: 349-60$.

40. Greenman $C$, Stephens $P$, Smith R, et al. Patterns of somatic mutation in human cancer genomes. Nature $2007 ; 446: 153-8$.

41. Katayama T, Imaizumi K, Manabe T, et al. Induction of neuronal death by $\varepsilon R$ stress in Alzheimer's disease. J Chem Neuroanat $2004 ; 28: 67-78$

42. Ozcan U, Cao $Q$, Yilmaz $\varepsilon$, et al. Endoplasmic reticulum stress links obesity, insulin action, and type 2 diabetes. Science $2004 ; 306: 457-61$.

43. Lipson KL, Fonseca SG, Ishigaki S, et al. Regulation of insulin biosynthesis in pancreatic beta cells by an endoplasmic reticulum-resident protein kinase IREl. Cell Metab $2006 ; 4: 245-54$.

44. Foufelle F, Ferré P. La réponse UPR : son rôle physiologique et physiopathologique. Med Sci (Paris) $2007 ; 23: 291-6$.

45. Barouki R. Stress oxydant et vieillissement. Med Sci (Paris) $2006 ; 22: 266-72$.

46. Bisbal C, Salehzada T. La RNase L, un acteur essentiel de la réponse cellulaire antivirale. Med Sci (Paris) $2008 ; 24: 859-64$

TIRÉS À PART

६. Chevet

\section{DEUX OUVRAGES INDISPENSABLES DE LA NOUVELLE COLLECTION PHARE :}

La callection a FEssentiel de limagerie Médicale s englobe tous les diagnostics les plus importants de chaque speiciabie clinique Les differents volumes de ta collection correspondent soit a une region anatomique, soit a une discipline medicale specifique.

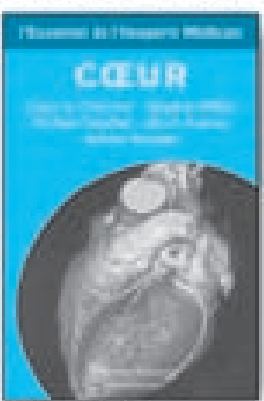

25 Neviler 2009 320 pases 260 illustration: Prix pobele Tre i 39 e
I'Essentiel de I'Imagerie Médicale : COEUR

\section{Par Claus D. CLAUSSEN}

Un livre pratique et très complet qui traite successivement des cardiopathies ischémiques, de l'insuffisance cardiaque, des valvulopathies, des myocardiopathies, des cardiopathies hypertensives, des tumeurs, des cardiopathies congénitales, des mala. dies des gros vaisseaux, des traumatismes thoraciques.

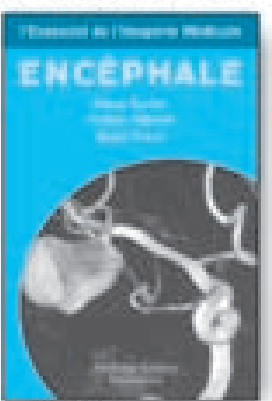

\section{5 sturier 2009} 320 paves 348 illistration: Pir astrations
I'Essentiel de I'lmagerie Médicale : ENCÉPHALE

\section{Par Klaus SARTOR}

Cet ouvrage, trè's exhaustif, expose toutes les pathologies observées en pratique clinique: Traumatismes ctàniens, pathologies inflammatoires, malformations vasculaires, accidents vasculaires cérébraux, pathologie tumorale, méningée, leuco-encéphalite, malformations congénitales, études post-opératoires, artefacts en IRM.

En vente dhez volve libroire specciolise, por correspondance ov sur notre ste muw medecine flommarion.com

\section{$x$

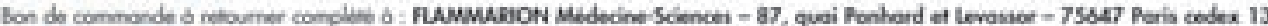

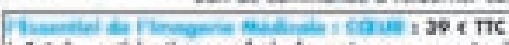

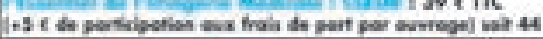

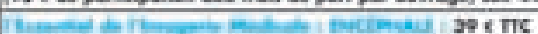

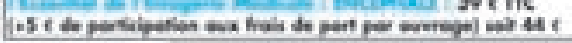
Nom / hinom:

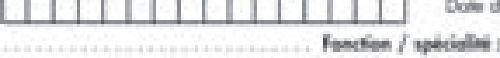
Tal 1 font

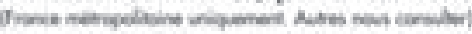

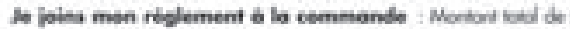

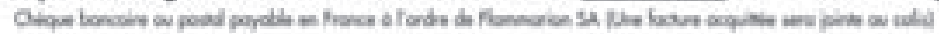

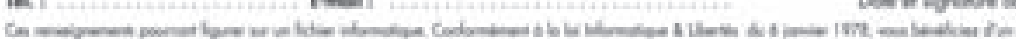

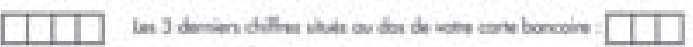

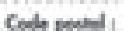
Absue : 